\title{
(Re-)Design of a Demonstration Model for a Flexible and Decentralized Cyber-Physical Production System (CPPS)
}

\author{
Georg Egger ${ }^{1, *}$, Erwin Rauch $^{2}$, Dominik T. Matt ${ }^{1,2}$ and Christopher A. Brown ${ }^{3}$ \\ ${ }^{1}$ Fraunhofer Italia Research s.c.a.r.l., Innovation Engineering Center (IEC), 39100 Bolzano, Italy \\ ${ }^{2}$ Faculty of Science and Technology, Free University of Bozen-Bolzano, Bolzano, 39100 Italy \\ ${ }^{3}$ Dept. of Mechanical Engineering, Worcester Polytechnic Institute, Worcester, 01609, MA, USA
}

\begin{abstract}
Cyber-physical production systems (CPPS) enable completely new possibilities in the factory of the future through the connectivity between the digital world and the physical production system. It has so far been difficult to transfer these advantages and concepts to students as well as professionals in a clear and practical oriented manner. The Fraunhofer Italia team has built a demonstration model for a flexible and decentralized CPPS system for showcase purposes. As part of an improvement of the existing system, Axiomatic Design (AD) was applied as the scientific design theory. Starting from the identification of the Customer Attributes, Functional Requirements were derived and the Design Parameters for the new design were defined by means of the AD decomposition and mapping process. In this paper, the application of AD for product improvement is described step-by-step based on the example of the redesign of the CPPS demonstration model.
\end{abstract}

\section{Introduction}

Cyber-Physical Systems (CPS) and other Industry 4.0 technologies are the enablers for new business models, which have the potential to be disruptive [1]. The term "Industry 4.0" is spoken of highly as a bright vision in research and industry to revolutionize production management and the factory of the future. After mechanization, electrification and computerization of industrial production we are now at the beginning of a new epoch in production, where web technology, intelligent automation as well as digitalization supports the development of CPS [2].

Many companies in various industries have reorganized their production in the recent past, following the principles of Lean Production [3, 4] or even taking advantage of novel production strategies such as Agile Manufacturing [5] and Mass Customization [6], and thereby increasing flexibility and achieving significant progress in productivity and in readiness for delivery [7].

New Industry 4.0 technologies and CPS enable completely new opportunities for the realization of highly efficient and responsive production systems for the production of individual products on demand. Industry 4.0 technologies facilitate the fabrication of customized products and thus, the concept of mass customization [8].

The majority of enterprises is still quite sceptical regarding the vision of Industry 4.0. In addition, large enterprises tend to feel better prepared than small enterprises. In other words, SMEs still show deficits compared to large enterprises [9].
Thus, Industry 4.0 represents a special challenge for businesses, but also for education. One possibility to train employees as well as students in Industry 4.0 technologies is learning factories. Learning factories can make a substantial contribution toward the understanding of Industry 4.0. Workplace-related scenarios can be mapped providing practical learning. This process enables participants to transfer learned knowledge directly to the own workplace [10]. In recent years, a large number of learning factories have been created for the purpose of knowledge transfer $[10,11,12]$. However, it is not always possible to train and show these emerging concepts directly in a real factory environment. Therefore, demonstration models are a popular alternative and complementary solution, where the concepts and technologies can be demonstrated and explained in a miniaturized way to employees and students.

The paper shows an Axiomatic Design (AD) based redesign of demonstration model of a flexible and decentralized Cyber-Physical Production Systems (CPPS). The demonstration model created by Fraunhofer Italia is intended to facilitate the knowledge transfer of Industry 4.0 concepts to project partners, industrial firms as well as students from schools and universities. The paper is structured as follows: after this introduction, the authors summarize the theoretical background on CPPS and demonstration models. Afterwards, the current design of the demonstration model is described in detail in section 3. In section 4, the authors show an AD based approach starting with the collection of the Customer Attributes (CAs) and defining the Functional

\footnotetext{
${ }^{*}$ Corresponding author: georg.egger@,fraunhofer.it
} 
Requirements (FRs). After this, they derive the Design Parameters (DPs) of the new model through an AD decomposition and mapping process. In section 5, they illustrate the redesigned concept for the demonstration model and close the paper with a brief summary and an outlook for future research activities.

\section{Theoretical background}

The theory section presents a brief overview and the state of the art regarding to CPPS as well as the purpose and use of demonstration models.

\subsection{Industry 4.0 and Cyber-Physical Production Systems (CPPS)}

The term cyber-physical system (CPS) stands for the continuously increasing presence of computing and communication capabilities in physical objects in the real world. The decision making process of such systems may be supported or handled autonomously by computational intelligence.

The concept of CPS has already been the subject of research for over 10 years [13], although first in other than production-related contexts. When the vision of Industry 4.0 was formulated [14], direct reference to CPS for production industry was made. CPS positively affected manufacturing in form of CPPS in process automation and control [15]. Since then, the terms Industry 4.0 and CPPS are used for the impact of the macro-trend digitalization on the production industry (see Fig. 1).

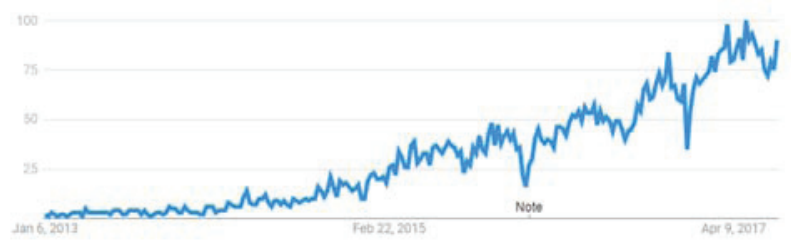

Fig. 1. Increasing (relative) popularity of the search term "Industry 4.0" on Google since 2013 (the term was particularly popular in Germany, Italy, Japan, India and the UK) [16]. The values indicate the search interest relative to the highest point in the chart in the specified period.

The vision of Industry 4.0 tries to respond to today's challenges like [14]:

- Demand for improved efficiency, especially in developed countries, in terms of productivity and energy efficiency,

- Trend towards individualisation and small batch sizes,

- Need to create horizontal value networks extending across businesses.

In order to achieve the goals, Industry 4.0 foresees 4 pillars [17]: Interconnection of humans and machines via the Internet, information transparency between the physical world and its virtual model, autonomous decisions at lowest possible level (decentralised), and finally, technical assistance in decision making and problem solving to humans to cope with the ever more complex CPPS. Across all pillars, standards, security, safety and human-machine-interfaces play a significant role.

The term Industry 4.0 is often being used by suppliers and governmental bodies, and on technical components that are sold by numerous suppliers. However, its practical use in factories is impeded by shortcomings in, for example, the lack of common standards for both horizontal and vertical integration, models for controlling complex structures, and qualified personnel [18]. Consequently, companies, especially small and medium sized enterprises (SMEs), are often forced to postpone the comprehensive adoption of the concepts of Industry 4.0.

\subsection{Industry 4.0 Labs and Demonstration Models}

Demonstration in a safe environment ("sandboxing") overcomes part of the obstacles in implementing Industry 4.0 concepts in production, because it does not affect the operational production. This encourages the experimental use of advanced methods for controlling productions and innovative technologies, and creates a breeding ground of regional innovation by training the involved people. If such an infrastructure is located in an independent institution like a Fraunhofer Institute, open to all interested companies, its effectiveness is maximised and a positive impact to the regional companies can be achieved.

These benefits were already recognised particularly in Germany, where application examples, laboratories and test centres are united through the network "Plattform Industrie 4.0" [19]. This platform counts almost 284 test examples for Industry 4.0. Such labs and application centres benefit from a favourable ecosystem, e.g., universities with relevant research focus, or suppliers of technologies. For example, in Kaiserslautern, where the German Research Centre for Artificial Intelligence (DFKI) partnered up with the local technical university and well-known companies such as Siemens, Bosch, SAP and several others, a supplierindependent Smart Factory lab for innovative technologies, control architectures and components, as well as a consulting services for interested companies was created [20]. The initiative fostered a successful ecosystem and soon several other companies participated in the initiative.

Demonstration is an important step towards the implementation of Industry 4.0 concepts in companies [20], where smart factory labs are an obvious step to make. The major R\&D challenges for demonstration models are listed here for a summary [20]:

- Context-adaptive and (at least partially) autonomous systems,

- Cooperative production systems,

- Identification and prediction of dynamical systems,

- Robust scheduling,

- Fusion of real and virtual systems,

- Human-machine symbiosis. 
In order to enhance usefulness, the ability to demonstrate the potential of Industry 4.0 to the interested audience to perform research in one or more of the fields listed above needs to be developed.

\section{Current design of the Demonstration Model}

The current model was built by a team of Fraunhofer Italia researchers and students to demonstrate the potentials of a cyber-physical production system in the factory of the future. The model has been presented the first time in public during the long night of research in September 2016 in the Free University of Bolzano. Mainly the model aims to demonstrate the following concepts of typical and modern factory of the future concepts to students and SMEs:

- Flexible Transport System

- Intelligent Workpiece Carrier

- Decentralized Control

- Digital Interconnection

- Efficient Human-Machine Interface.

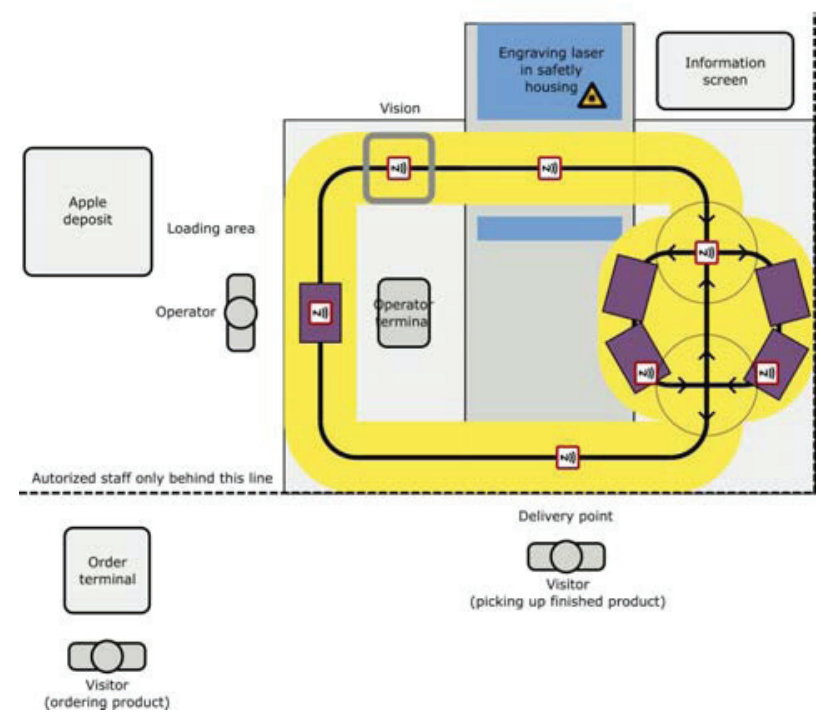

Fig. 2. System Architecture of the demonstration model. Yellow areas around the black tape indicate the boundaries of vehicle navigation; purple rectangles represent the dimensions of the vehicles; dotted circles indicate the manoeuvring area around crossings, which should be respected by the other vehicles.

Figure 2 and 3 illustrate the system architecture and the real demonstration model consisting of a:

- Laser engraving head

- Safety housing (custom design)

- Fume extraction unit

- Air compressor

- NFC (near field communication) pads

- Vehicles for product transport.

The vehicles presented in Figures 3 and 4 consist of a commercial robotic platform with 4 DC motors, an Arduino-compatible controller board, an internally developed apple-spin mechanism (apple symmetry axis aligns to spin axis), a custom built line sensor with 8 analogue sensors, an IR distance sensor for collision avoidance, an apple-eject mechanism, and a control algorithm (line follower) with routing capability and the ability to detect and take crossings. In addition, a user interface was developed to get orders from a computer terminal. Visitors may enter their name and a personal message to be engraved on the apple. After confirmation through an individual RFID card or tag. Before the order is executed, the message needs approval by a human operator. When ready, a screen informs the visitor to pick up his apple. Visitors need to present their RFID card in order to start the delivery of the apple.

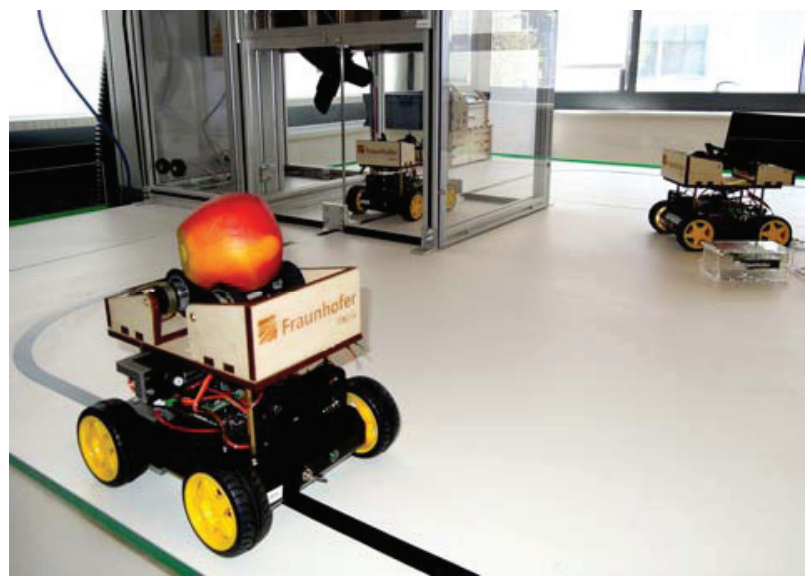

Fig. 3. CPPS demonstration model.

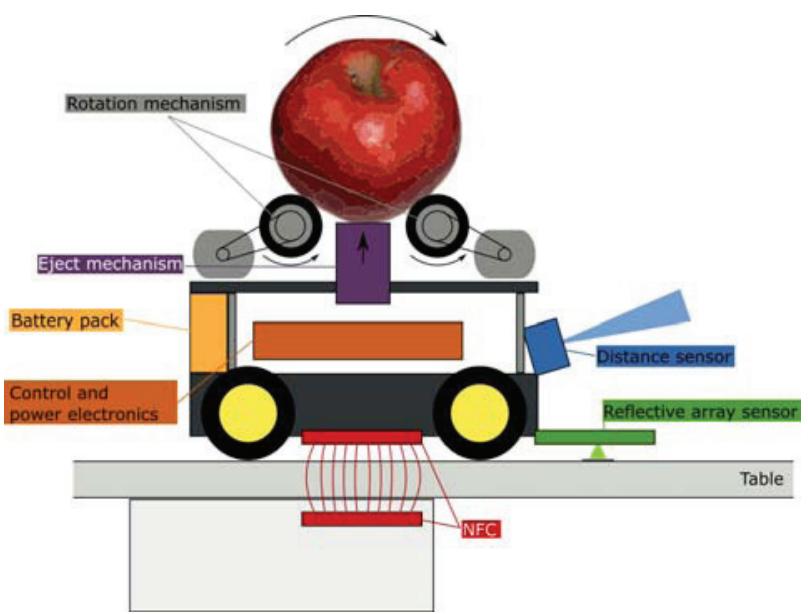

Fig. 4. Semi-autonomous vehicle - schematic view with major subsystems including (near-field communication) NFC connection between vehicle and stationary parts (pads).

\section{Re-design of the CPPS Demonstration Model using Axiomatic Design (AD)}

After the first successes of the demonstration model at the Long Night of Research, it should now be presented to a larger audience by planning to disassemble the model and transport it in an efficiently way and to build it up on-site at schools, SMEs, universities or other facilities or events. For this purpose, the design is revised, further functions should be integrated and at the same time analysed critically regarding design flaws and possible improvements. For this purpose, a workshop was held in the presence of an AD expert. 
AD was developed by Nam P. Suh in the mid-1970s in the pursuit of developing a scientific, generalized, codified, and systematic procedure for design. The scientific theory get its name from two axioms in AD that have to be respected. The first is the Independence Axiom: Maintain the independence of the functional elements, i.e., avoid coupling in the system (e.g., avoiding dependencies between the DPs and other FRs). The second is the Information Axiom: Minimize the information content: select the solution with the least information content, i.e., that has the highest probability of success [21]. In order to apply these axioms, parallel functional and physical hierarchies are constructed, the latter containing the physical design solutions. The impact of $\mathrm{AD}$ is that the designer learns how to construct large design hierarchies quickly that are more structured, thus freeing more time for mastering applications [22].

In the initial workshop, requirements and so-called CAs were collected. Based on this input, FRs and Cs are defined and design parameters for a redesign were derived in an AD top-down decomposition and mapping process.

\subsection{Workshop to define Customer Attributes (CAs)}

In the workshop, the research team collected the requirements and needs and categorized them in the following groups [23]:

a) Constraints (Cs) are usually hard limits or values (minimum, maximum, between).

b) Functional Requirements (FRs) help the designer in the determination of the sub-levels requirements and related design solutions. They should be independent from each other to comply with axiom one, reduce complexity of the system design and are characterizing the functional needs of the artefact.

c) Non-Functional Requirements (non-FRs) focus on "how" the artefact should be (usually "should be" together with an adjective) and can influence functional requirements.

The following CAs could be identified (see Table 1):

Table 1. Customer Attributes (CAs).

\begin{tabular}{|c|c|c|c|}
\hline Nr. & Customer Attribute & $\begin{array}{l}\text { C, FR, } \\
\text { n-FR }\end{array}$ & Existing \\
\hline $\mathrm{CA}_{1}$ & $\begin{array}{c}\text { Demonstrate intelligent and } \\
\text { decentralized control of } \\
\text { individualized production } \\
(\text { lot size } 1)\end{array}$ & $\rightarrow \mathrm{FR}_{4}$ & Yes \\
\hline $\mathrm{CA}_{2}$ & $\begin{array}{l}\text { Permit rapid scaling of the } \\
\text { capacity up and down } \\
\text { automatically }\end{array}$ & $\rightarrow \mathrm{FR}_{2}$ & In part \\
\hline $\mathrm{CA}_{3}$ & $\begin{array}{l}\text { The product should have a } \\
\text { local link and allow } \\
\text { marketing of the region of } \\
\text { South Tyrol }\end{array}$ & $\stackrel{\overrightarrow{\mathrm{n}}-\mathrm{FR}_{1}}{ }$ & Yes \\
\hline $\mathrm{CA}_{4}$ & $\begin{array}{l}\text { The model should be } \\
\text { realized with students for } \\
\text { training purpose }\end{array}$ & $\stackrel{\overrightarrow{\mathrm{n}}-\mathrm{FR}_{2}}{ }$ & Yes \\
\hline $\mathrm{CA}_{5}$ & $\begin{array}{l}\text { The demonstration model } \\
\text { should show a complete }\end{array}$ & $\begin{array}{l}\overrightarrow{7} \\
\mathrm{n}-\mathrm{FR}_{3}\end{array}$ & In part \\
\hline
\end{tabular}

\begin{tabular}{|c|c|c|c|}
\hline Nr. & Customer Attribute & $\begin{array}{l}\text { C, FR, } \\
\text { n-FR }\end{array}$ & Existing \\
\hline & production process & & \\
\hline $\mathrm{CA}_{6}$ & $\begin{array}{l}\text { The maximal budget for } \\
\text { realization are 20,000 Euro }\end{array}$ & $\rightarrow \mathrm{C}_{1}$ & Yes \\
\hline $\mathrm{CA}_{7}$ & $\begin{array}{l}\text { Modularity in order to reuse } \\
\text { the single components also } \\
\text { in other educational or } \\
\text { research settings. }\end{array}$ & $\rightarrow \mathrm{C}_{3}$ & Yes \\
\hline $\mathrm{CA}_{8}$ & $\begin{array}{l}\text { Maximum space for the } \\
\text { demonstration model is } 6 \mathrm{~m}^{2}\end{array}$ & $\rightarrow \mathrm{C}_{2}$ & Yes \\
\hline $\mathrm{CA}_{9}$ & $\begin{array}{l}\text { The demonstration model } \\
\text { needs to be movable to use it } \\
\text { in different events and } \\
\text { facilities }\end{array}$ & $\rightarrow \mathrm{C}_{4}$ & In part \\
\hline $\mathrm{CA}_{10}$ & $\begin{array}{l}\text { Safety for user and visitors } \\
\text { has to be guaranteed }\end{array}$ & $\rightarrow \mathrm{FR}_{3}$ & Yes \\
\hline $\mathrm{CA}_{11}$ & $\begin{array}{l}\text { Allow interaction between } \\
\text { visitor and the CPPS demo } \\
\text { model }\end{array}$ & $\rightarrow \mathrm{FR}_{3}$ & Yes \\
\hline $\mathrm{CA}_{12}$ & Traceability of orders & $\rightarrow \mathrm{FR}_{4}$ & In part \\
\hline $\mathrm{CA}_{13}$ & $\begin{array}{l}\text { Quality check of the order } \\
\text { before delivery }\end{array}$ & $\rightarrow \mathrm{FR}_{3}$ & In part \\
\hline $\mathrm{CA}_{14}$ & $\begin{array}{l}\text { Delocalisation of order entry } \\
\text { (e.g. on mobile devices) }\end{array}$ & $\rightarrow \mathrm{FR}_{3}$ & Yes \\
\hline $\mathrm{CA}_{15}$ & $\begin{array}{l}\text { Demonstration of advanced } \\
\text { industrial robotics in the } \\
\text { CPPS }\end{array}$ & $\rightarrow \mathrm{FR}_{1}$ & No \\
\hline $\mathrm{CA}_{16}$ & $\begin{array}{l}\text { Experience the production } \\
\text { from the point of view of the } \\
\text { product }\end{array}$ & $\rightarrow \mathrm{FR}_{5}$ & No \\
\hline
\end{tabular}

Table 1 shows also if the requirements are fully fulfilled or only partly fulfilled (improvable) in the current demonstration model or if they are new.

\subsection{Definition of high-level Functional Requirements (FRs) and Design Parameters (DPs)}

The highest level of FRs and DPs are:

$\mathrm{FR}_{0} \quad$ Demonstrate Industry 4.0 concepts for CPPS in a practical way.

$\mathrm{DP}_{0}$ Demonstration Model for a flexible and decentralized CPPS.

Based on the outcome of the initial workshop the following Cs for the (re)design were defined:

$\mathrm{C}_{1} \quad$ Maximum total budget of 20,000 Euro for new investments.

$\mathrm{C}_{2} \quad$ Maximum space of 6 square meters.

$\mathrm{C}_{3} \quad$ Modular structure with standard components.

$\mathrm{C}_{4} \quad$ Mobility of the demo model for flexible use.

Further the following n-FRs were defined:

n-FR 1 Products should have a local link with the region of South Tyrol.

$\mathrm{n}-\mathrm{FR}_{2}$ The model should be realized and run with the support of students for training purpose.

$\mathrm{n}-\mathrm{FR}_{3}$ The demonstration model should show a complete production process. 
Finally the remaining CAs were associated to high-level Functional Requirements deriving Design Parameters:

$\mathrm{FR}_{1} \quad$ Apply advanced industrial robotics in the CPPS.

$\mathrm{FR}_{2}$ Allow automatic scaling of capacity up and down.

$\mathrm{FR}_{3}$ Ensure safety during operation of personnel, visitors and equipment.

$\mathrm{FR}_{4} \quad$ Event-based dynamic control and monitoring of a production line for mass customized products.

$\mathrm{FR}_{5} \quad$ Visualize the production from the point of view of the product.

$\mathrm{DP}_{1}$ Lightweight robot and vision system for bin picking at the loading station.

$\mathrm{DP}_{2}$ Buffer for waiting vehicles and automatic call.

$\mathrm{DP}_{3}$ Safe user-interface between user/visitor and the cyber-physical system

$\mathrm{DP}_{4}$ Intelligent and autonomous vehicles driven by a decentralized control architecture.

$\mathrm{DP}_{5}$ Camera system on the work piece carrier.

The design matrix on the first level is decoupled and shows the dependencies between the solutions (DPs) and the functional requirements (FRs):

$$
\left\{\begin{array}{l}
F R_{1} \\
F R_{2} \\
F R_{3} \\
F R_{4} \\
F R_{5}
\end{array}\right\}=\left[\begin{array}{ccccc}
X & 0 & 0 & 0 & 0 \\
X & X & 0 & 0 & 0 \\
0 & 0 & X & 0 & 0 \\
X & 0 & X & X & 0 \\
0 & 0 & 0 & X & X
\end{array}\right]\left\{\begin{array}{l}
D P_{1} \\
D P_{2} \\
D P_{3} \\
D P_{4} \\
D P_{5}
\end{array}\right\}
$$

$\mathrm{DP}_{1}$ has also influence on $\mathrm{FR}_{2}$ and $\mathrm{FR}_{4}$ and $\mathrm{DP}_{3}$ has influence on $\mathrm{FR}_{4}$. The same holds for $\mathrm{DP}_{4}$ and $\mathrm{FR}_{5}$. These off-diagonal interactions, showing a coupling of DPs and other FRs, are explained more in detail in Section 4.3.6 on the different levels. Figure 5 shows the FR-DP tree of the highest hierarchical levels.

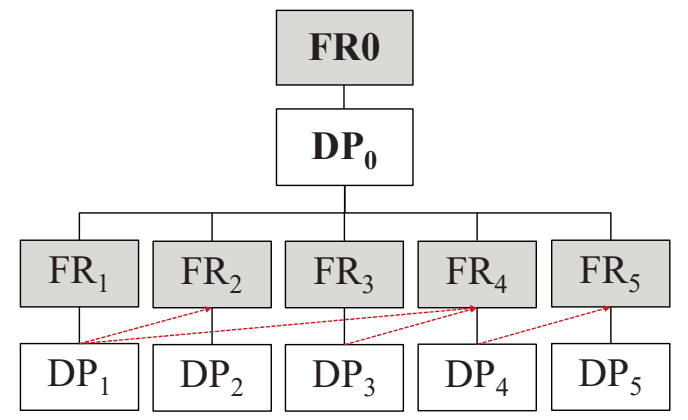

Fig. 5. Highest-level FR-DP tree.

\subsection{Top-Down decomposition and mapping process}

The decomposition process of top-level FRs and DPs aims to transform the abstract requirements into more concrete parameters that are close to the daily practice and therefore relevant for implementation. The FR-DP pairs on the highest hierarchical level represented in Figure 5 are a starting point for the top-down decomposition and mapping process in AD. The decomposition is performed separately for each of the
FR-DP pairs shown in Figure 5 to obtain a better understanding of the process.

\subsubsection{FR $1-D P_{1}-$ Industrial Robotics}

Industrial robotics can be integrated using a lightweight robot for picking apples from the container and loading them on the vehicles. Starting from $\mathrm{FR}_{1}$, further FRs and DPs of the successive hierarchical level can be defined as follows:

$\mathrm{FR}_{1.1}$ Localize and identify apple for flexible feeding.

$\mathrm{FR}_{1.2}$ Grasping of sensitive products.

$\mathrm{DP}_{1.1}$ Lightweight robot combined with vision system for bin picking of the apple.

$\mathrm{DP}_{1.2}$ Flexible gripper for complex and sensitive products.

The design matrix shows an uncoupled design:

$\left\{\begin{array}{l}F R_{1.1} \\ F R_{1.2}\end{array}\right\}=\left[\begin{array}{ll}X & 0 \\ 0 & X\end{array}\right]\left\{\begin{array}{l}D P_{1.1} \\ D P_{1.2}\end{array}\right\}$

For flexible feeding, the research team can use existing equipment. A mobile station with a mounted UR3 lightweight robot combined with a camera system allows flexible feeding without additional investments. For grasping the apples, a flexible and sensitive gripper is required to avoid damages to the product.

\subsection{2 $F R_{2}-D P_{2}-$ Scalability}

Scalability is a major requirement of modern production systems. While in the current demonstration model the number of vehicles is fixed, a buffer should be created in the redesigned demonstration model. All other stations (laser engraving and robotized loading station) are sufficiently rapidly scalable in their performance. Vehicles not needed in periods with low demand can be parked in the buffer to reduce energy consumption in the system, while they are called automatically, when demand is rising. $\mathrm{FR}_{2}$ can be decomposed further as follows:

$\mathrm{FR}_{2.1}$ Provide function for rapid scaling down

$\mathrm{FR}_{2.2}$ Provide function for rapid scaling up to guarantee capacity for higher demand.

$\mathrm{DP}_{2.1}$ Crossing points with NFC pads to detect vehicle direction for ejection of the vehicles in a buffer line.

$\mathrm{DP}_{2.2}$ Buffer line with sufficient length to guarantee the needed capacity.

The design matrix shows an uncoupled design:

$\left\{\begin{array}{l}F R_{2.1} \\ F R_{2.2}\end{array}\right\}=\left[\begin{array}{ll}X & 0 \\ 0 & X\end{array}\right]\left\{\begin{array}{l}D P_{2.1} \\ D P_{2.2}\end{array}\right\}$

To realize the buffer line some more NFC pads have to be made for integration in the demonstration model. In addition, the dimensions (length) of the buffer line shall be defined according to the maximum number of 
vehicles, in order to guarantee the expected performance of the model during visitor presentations.

\subsection{3 $F R_{3}-D P_{3}$ - Interaction User-System}

To increase the attention and know-how transfer during visitor presentations the model should allow for interaction between the visitor/user and the CPPS. In the current demonstration model, visitors may create an individual order for writing an individual text on an apple by using a desktop station or their smartphone. Any order requires approval by a supervisor (in order to avoid inappropriate texts). To avoid injuries and malfunctions of the production equipment, the visitor cannot touch the vehicles or any other stations, and the finished apple ejects to a withdrawal tray, where the visitor may grasp it without interfering with the vehicle itself. In the current system, a quality check is missing for the simulation of a complete production process. Further, in addition to the visualization on a screen, the result of the quality check as well as the availability of the apple at the delivery station should be sent to the visitor via app. Thus, this new functions should be integrated in the redesigned demonstration model. $\mathrm{FR}_{3}$ can be decomposed in the following lower level FRs and DPs:

$\mathrm{FR}_{3.1}$ Prevent direct intervention by the user in unsafe areas

$\mathrm{FR}_{3.2}$ Create individual order in-situ or remotely.

$\mathrm{FR}_{3.3}$ Compliance check of incoming orders.

$\mathrm{FR}_{3.4}$ Quality check after processing.

$\mathrm{FR}_{3.5}$ Inform user/visitor about the order progress.

$\mathrm{DP}_{3.1}$ Separation of unsafe areas (e.g. through acrylic glass screen).

$\mathrm{DP}_{3.2}$ Order creation (individual text on the apple) at the order terminal or via smartphone (app).

$\mathrm{DP}_{3.3}$ Approval by supervisor on a monitor screen.

$\mathrm{DP}_{3.4}$ Camera system at laser engraving station to compare the result with the text in the order.

$\mathrm{DP}_{3.5}$ Notification to the visitor after laser engraving station and at delivery station.

The design matrix shows a decoupled design:

$$
\left\{\begin{array}{l}
F R_{3.1} \\
F R_{3.2} \\
F R_{3.3} \\
F R_{3.4} \\
F R_{3.5}
\end{array}\right\}=\left[\begin{array}{ccccc}
X & 0 & 0 & 0 & 0 \\
0 & X & 0 & 0 & 0 \\
0 & X & X & 0 & 0 \\
0 & 0 & 0 & X & 0 \\
0 & 0 & 0 & X & X
\end{array}\right]\left\{\begin{array}{l}
D P_{3.1} \\
D P_{3.2} \\
D P_{3.3} \\
D P_{3.4} \\
D P_{3.5}
\end{array}\right\}
$$

In addition to the diagonal interactions, $\mathrm{DP}_{3.2}$ also influences $\mathrm{FR}_{3.3}$. This influence occurs, because a remote order creation via smartphone in $\mathrm{DP}_{3.2}$ allows visitors to write a text on the apple. To avoid non-suitable text writing during demonstrations the text needs to be checked manually for its compliance $\left(\mathrm{FR}_{3.3}\right)$. Furthermore also $\mathrm{DP}_{3.4}$ shows an influence on $\mathrm{FR}_{3.5}$. The result of the quality check at the laser engraving station $\left(\mathrm{DP}_{3.4}\right)$ determines, if information can be transmitted and visualized to the user/visitor $\left(\mathrm{FR}_{3.5}\right)$.
While $\mathrm{DP}_{3.1}$ to $\mathrm{DP}_{3.3}$ were already part of the original model, the other two DPs shall be implemented in the redesigned demonstration model.

\subsubsection{FR $\mathrm{F}_{4}-\mathrm{DP}_{4}-$ Decentralized Control}

The decentralized control as well as the traceability of mass customized products in the demonstration model is currently solved by the use of intelligent (NFC technology) and autonomous vehicles. $\mathrm{FR}_{4}$ can be decomposed in the following lower level FRs and DPs:

FR $_{4.1}$ Vehicles shall be aware of their position and communicate with the CPPS.

$\mathrm{FR}_{4.2}$ Bring mass customized products decentralized to their next processing station.

$\mathrm{DP}_{4.1}$ NFC technology for both communication and location awareness.

$\mathrm{DP}_{4.2}$ Autonomously navigating vehicles with their own drive, routing capability and controller for every work piece carrier.

The design matrix shows an uncoupled design:

$\left\{\begin{array}{l}F R_{4.1} \\ F R_{4.2}\end{array}\right\}=\left[\begin{array}{ll}X & 0 \\ 0 & X\end{array}\right]\left\{\begin{array}{l}D P_{4.1} \\ D P_{4.2}\end{array}\right\}$

The choice for NFC is motivated by the fact that it provides superior robustness against electromagnetic disturbances typical for an industrial production environment and the well-defined range of operation, which allows its use not only for communication purposes but also for unambiguous position detection. Same also for the modular vehicles built with standard components and hence there are no design changes in this FR-DP pair.

\subsubsection{FR $R_{5}-D P_{5}-$ User Experience}

The demonstration model should allow the visitor to follow the steps in the production process from the point of view of the product. In the current demonstration model this function was not integrated. There is no need to decompose this FR-DP pair any further, as $\mathrm{DP}_{5}$ can be implemented through a standard camera mounted on the vehicle. The livestream of the camera shall be transmitted to the information screen and allows the visitors following the production process from loading the apple, rotating the apple in the right position, laser engraving and ejection in the delivery station.

\subsubsection{Overall design matrix and summary}

The decomposition and mapping process helped the research team to better structure the requirements and to derive systematically the physical design solutions (DPs) without increasing complexity of the design. In developing the final design concept the constraints identified in section 4.2 shall be respected. Figure 6 summarizes the overall design matrix at first and second level in the Axiomatic Design software Acclaro DFFS. 
As shown in the decoupled first level design matrix in equation (1) some DPs influence other FRs. The overall design matrix in Figure 6 explains this influence in the lower levels. The maximum performance of the selected industrial lightweight robot in $\mathrm{DP}_{1.1}$ determines also the maximum length of the buffer line to scale up the capacity of the system $\left(\mathrm{FR}_{2.2}\right)$. Further, $\mathrm{DP}_{1.1}$ has influence on $\mathrm{FR}_{4.1}$, as the industrial robot needs information on the position of vehicles in order to pick the apple and place it on the vehicles. Also $\mathrm{DP}_{3.5}$ is affecting $\mathrm{FR}_{4.1}$, as the status notification at the delivery station needs position data of the vehicles. The last offdiagonal interaction is between $\mathrm{DP}_{4.1}$ as well as $\mathrm{DP}_{4.2}$ and FR5. Both DPs (NFC as communication technology and the use of autonomously navigating vehicles) influence the possibilities to visualize the production from the point of view of the product.

The demo model is built up in a modular way, costs less than $20 \mathrm{k} €$ and occupies less than $6 \mathrm{~m}^{2}$. Furthermore, the n-FR are fulfilled: with bin picking and final quality control a complete production process of a local product is simulated and the demo model will be realized with the support of students at Fraunhofer Italia.

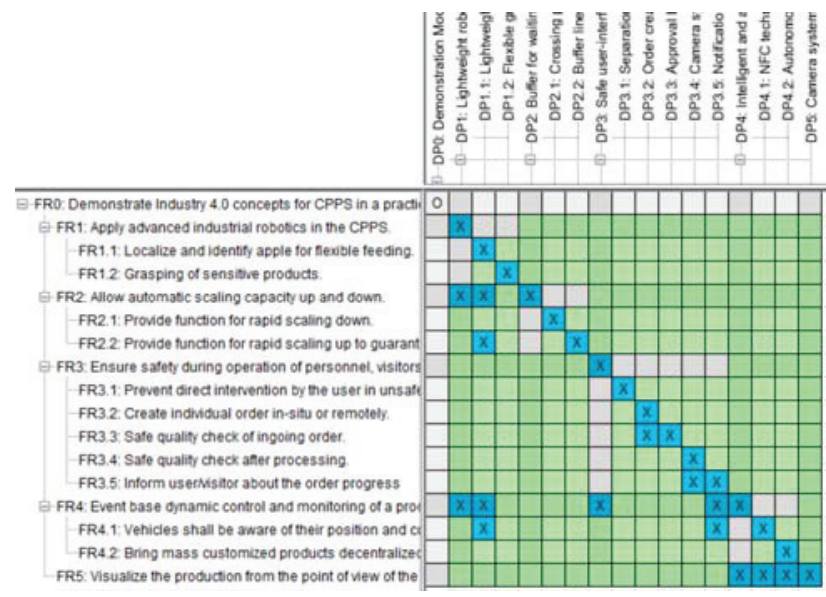

Fig. 6. Summarizing first and second level design matrix.

\section{Concept of the redesigned and improved CPPS Demonstration Model}

The analysis in section 4 revealed and improved a few weaknesses of the original demonstrator design, such as:

- Operation of the demonstrator required at least two people: One person is required for the actual operation in the reserved area and one for interacting with the visitors. This aspect is improved by inserting a bin-picking robotic arm in the layout, in line with $\mathrm{DP}_{1.1}$.

- The manual placement of the apples did not require the rotation mechanism, if the operator oriented the apple correctly, as he loads the vehicle. The binpicking gripper (result of $\mathrm{DP}_{1.2}$ ) likewise disposes of such functionality, and consequently a holding cup substitutes the now obsolete rotation mechanism on the vehicles.

- Vehicles could not be automatically added to the production process for rapid scalability, i.e., immediate, decentralized adjustment of the production rate; this had to occur manually and was relying on the operator recognizing the current demand. As suggested by $\mathrm{DP}_{2}$ a dedicated buffer line improves this situation. The required areas are obtained by re-designing the available board area. In the back, also the lateral boards now extend to the full length and the track layout considers the central area too. As a positive side effect, the overall capacity of the demonstrator was increased.

- In the waiting queues, empty and occupied vehicles were mixed in the two parking lanes. This not only violates the independence axiom, but also impedes an independent management of the vehicles required for loading area and delivery point, which resulted in unnecessarily frequent relocations of the vehicles and complex queue management.

- The table was not designed for optimal mobile use. The setup required additional structure elements to carry the tables and the cabling underneath did not allow quick and reliable setup. In line with $\mathrm{C}_{3}$ the tables were put on their own support structure and equipped with wheels. Further, the boards are hold together with two wing nuts, which do not even require tools for mounting and dismounting. Similarly, each board is pre-wired and the electrical interconnection of all low voltage systems can be realized with RJ45 patch cables from board to board. While a field bus interconnected the NFC pads, other low-level I/O's were excluded from that system, resulting in a heterogeneous wiring and unhandy complexity. New developed devices on the field bus substituted these non-standard components. Also, on underneath the boards, the mechanical placement of the field bus devices underwent unification process. Whereas previously each device was screwed into a particular position, rails were installed accommodating the field bus components. Now a displacement along the rails is easily possible for all components.

- The vehicles and the NFC pads have been subject of continuous improvement since the public presentation of the demonstrator. The field bus system and the associated communication protocol were continuously expanded. The NFC library was completely rewritten for improved reliability and usability. Also, the precision of the line follower algorithm was improved significantly reducing lateral motion.

- As one of the major goals of the demonstrator is to reach public attention for our research, also the nonfunctional aspects deserves attention, such as an attractive presentation. Previously the information screen merely informed the visitors when product were ready and showed videos from a fixed camera inside the laser housing - the latter mainly due to safety concerns. It turned out that the videos attracted much attention, though vehicles rested only briefly inside the laser. As suggested by $\mathrm{DP}_{5}$ the presentational aspect improves significantly by transmitting live video from a camera mounted on a vehicle. The resources on the vehicles previously 
used for the rotational mechanism may now be conveniently used for video transmission.

Most of the improvements are reflected in the layout of the demonstrator, which is illustrated in Figure 7.

In the redesigned demonstration model there is a clear distinction between active waiting for jobs (green), active waiting for delivery (purple) and standby in the buffer line (blue) vehicles. The loading mechanism not only grips the apples and places them on the vehicles, but also aligns them so that the rotation mechanism of the vehicles becomes obsolete. Hence, the vison process is now part of the loading. The operator is relieved from repetitive loading and may assist visitors. In addition, visitor may place orders remotely.

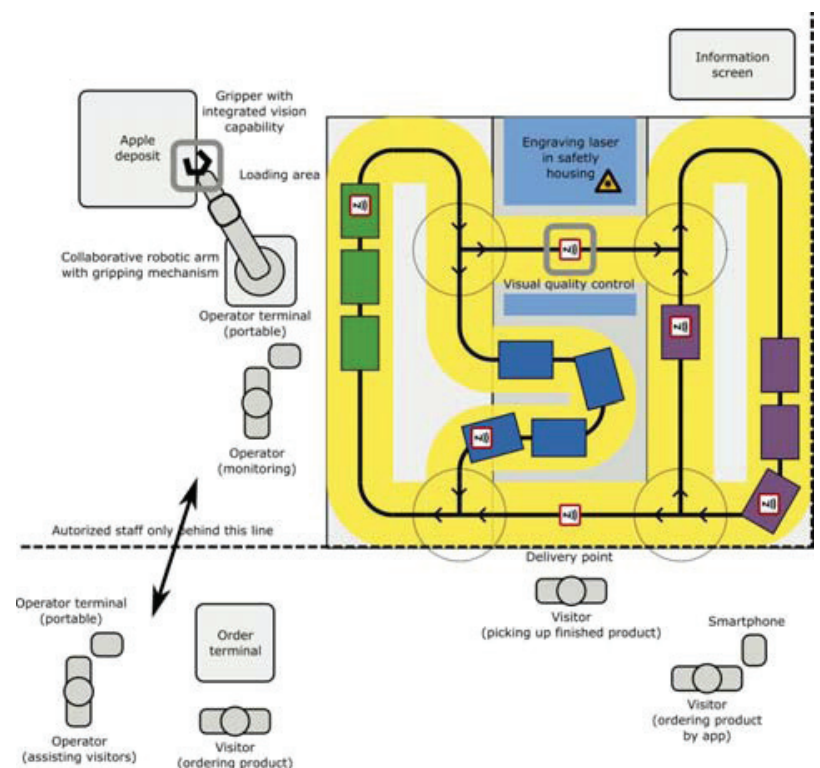

Fig. 7. System Architecture of the redesigned demonstration model.

\section{Conclusion and outlook for future research}

The present research activity has shown that axiomatic design is a powerful tool not only for new designs, but also for analysing and improving existing designs. It helped particularly in sharpening the focus on the original objectives, which during the implementation phase of the demonstrator got fuzzy. Furthermore, the systematic exercise of applying axiomatic design theory identified also gaps in the previous design, like e.g. the lack of quality control, missing buffer line, redundancy in functions (rotation mechanism, manual placement). Overall, the demonstrator benefits significantly from the activity: Its capacity increased just by changing the topology of the tracks, at the same time the control algorithms are simplified and the presentational quality improves by including also bin-picking robotic arm and live videos directly from the vehicles. Finally, the demonstrator gained in efficiency, as less people are required for operation and setup.

There remain some points for improvement:

- The overall weight of the demonstrator is unchanged, which hinders its portability. Particularly the laser (safety housing, fume extractor unit and compressor) is massive and in future lighter alternatives may be explored.

- The reliability of the vehicle motion control turned out to be limited without position feedback, as the wheels slip on the surface, which increases with usage. The use of inertial measurement units could improve the accuracy of movement particularly at crossings.

- When developing the updated setup, a variety of possible topologies was found. Without proper means of simulating their performance, the authors had to estimate the performance based on their experience. A quantitative confirmation of this result, as well as exploration of other possible topologies (e.g. a full mesh topology, Figure 8) is of interest to the authors and may be addressed in future work.

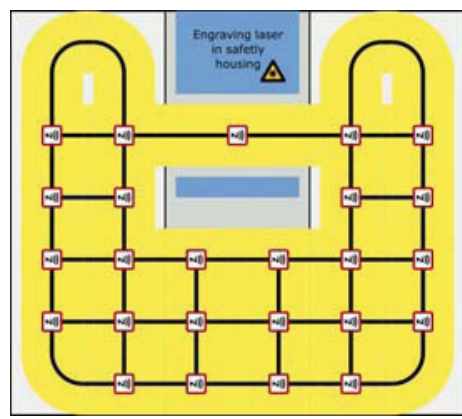

Fig. 8. Full mesh topology allowing dynamic reorganisation of the layout at the cost of several NFC pads. Its benefit and the potential reduction shall be analysed with adequate means of simulation in future.

The results of this research activity are applicable also to ongoing research projects, such as "DeConPro2, which shares many of the objectives of the demonstrator. The focus is on decentralized control, with realization of a model factory with industrial-grade components.

In the research project "SME 4.0", two work packages will develop design concepts for highly adaptable and intelligent CPPS for SME. As the working title already reveals, research focuses on the development of new concepts and the adaptation of existing approaches that are especially suitable for SME.
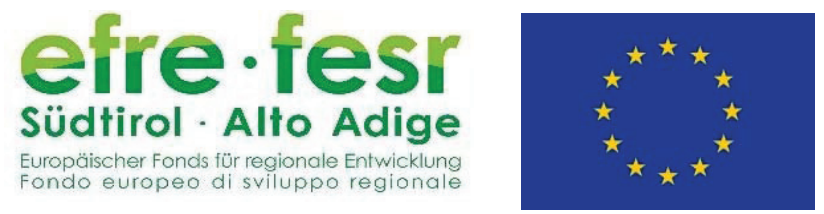

This research is part of actual research activities in the project DeConPro (Decentralized control of production processes in the factory of the future) and in the project SME 4.0 (Industry 4.0 for SMEs). The first has received funding from the European Regional Development Fund ERDF 2014-2020 under the grant agreement with project code FESR1017 (CUP B56J16001730001) and the second from the European Union's Horizon 2020 research and innovation program under the Marie Skłodowska-Curie grant agreement No 734713. 


\section{References}

1. E. Rauch, S. Seidenstricker, P. Dallasega, R. Hämmerl, Collaborative Cloud Manufacturing: Design of Business Model Innovations Enabled by Cyberphysical Systems in Distributed Manufacturing Systems, Journal of Engineering, Article ID 1308639, 12 pages (2016).

2. S.P.A. Datta, Dynamic socio-economic disequilibrium catalyzed by the Internet of Things. Available

from http://dspace.mit.edu/bitstream/handle/1721.1/86935 /Dynamic\%20Socio-

Economic\%20Disequilibrium\%20_\%20Shoumen\% 20Datta.pdf? sequence $=226$ [Accessed 06th February 2016], pp. 1-9, (2014)

3. D.T. Matt, E. Rauch, Implementing Lean in Engineer-to-order manufacturing - Experiences from a ETO Manufacturer, in: Modrák, V.; Semančo, P. (Eds.), Handbook of Research on Design and Management of Lean Production Systems, Hershey, IGI Global, 148-172, (2014).

4. N.V.K., Jasti, R. Kodali, Lean production: literature review and trends. International Journal of Production Research, 53(3), 867-885, (2015).

5. D.T. Matt, E. Rauch, Design of a scalable modular production system for a two-stage food service franchise system, International Journal of Engineering Business Management, 32, 4 (2012).

6. B. Stump, F. Badurdeen, Integrating lean and other strategies for mass customization manufacturing: a case study, Journal of Intelligent manufacturing, 23, 1, 109-124, (2012).

7. M. Brettel, N. Friederichsen, M. Keller, M. Rosenberg, How Virtualization, Decentralization and Network Building Change the Manufacturing Landscape: An Industry 4.0 Perspective, International Journal of Mechanical, Aerospace, Industrial, Mechatronic and Manufacturing Engineering, 8, 1, 37-44, (2014).

8. D.T. Matt, E. Rauch, Designing assembly lines for mass customization production systems, in $\mathrm{V}$. Modrák, D. Mourtzis (Eds.), Mass Customized Manufacturing: Theoretical Concepts and Practical Findings, Boca Raton, CRC Press Francis \& Taylor, 15-35, (2017).

9. L. Sommer, Industrial revolution-industry 4.0: Are German manufacturing SMEs the first victims of this revolution? Journal of Industrial Engineering and Management, 8, 5, 1512-1532, (2015).

10. C. Prinz, F. Morlock, S. Freith, N. Kreggenfeld, D. Kreimeier, B. Kuhlenkötter, Learning Factory Modules for Smart Factories in Industrie 4.0, Procedia CIRP, 54, 113-118, (2016).

11. D.T. Matt, E. Rauch, E., P. Dallasega, Mini-factorya learning factory concept for students and small and medium sized enterprises, Procedia CIRP, 17, 178183, (2014).
12. C. Faller, D. Feldmüller, Industry 4.0 learning factory for regional SMEs, Procedia CIRP, 32, 8891, (2015).

13. R. Rajkumar, I. Lee, L. Sha, J. Stankovic, CyberPhysical Systems: The Next Computing Revolution, Design Automation Conference, June 2010, 731736, (2010).

14. H. Kagermann, W. Wahlster, J. Helbig, Securing the future of German manufacturing industry, Recommendations for implementing the strategic initiative INDUSTRIE 4.0 Final report of the Industrie 4.0 Working Group, acatech, (2013).

15. L. Monostori, Cyber-physical production systems: roots, expectations and R\&D challenges, Procedia CIRP 17, 9-13, (2014).

16. Industry 4.0, Available from https://rends.google.com/trends/explore?q=Industry $\% 204.0$ [Accessed 24th July 2017].

17. M. Hermann, T. Pentek, B. Otto, Design Principles for Industrie 4.0 Scenarios, 49th Hawaii International Conference on System Sciences (HICSS), 3928-3937, (2016).

18. K. Zhou, T. Liu, L. Zhou, Industry 4.0: Towards future industrial opportunities and challenges, Proceedings of 12th International Conference on Fuzzy Systems and Knowledge Discovery (FSKD), 2147-2152, IEEE, (2015).

19. Labs Network Industry 4.0, Available from http://lni40.de/ [Accessed 10th April 2017].

20. D. Zuehlke, SmartFactory-Towards a factory-ofthings, Annual Reviews in Control 34, 129-138, (2010).

21. N.P. Suh, Axiomatic Design - Advances and Applications. New York, Oxford University Press, (2009).

22. C.A. Brown, Teaching axiomatic design to engineers-Theory, applications, and software, Journal of Manufacturing Systems, 24, 3, 186-195, (2005).

23. M.K. Thompson, A Classification of Procedural Errors in the Definition of Functional Requirements in Axiomatic Design Theory, Proceedings of the 7th International Conference on Axiomatic Design, Worcester, MA, 107-112, (2013). 